\title{
Contaminación cruzada por el uso de tubos de resinas compartidos en las clínicas odontológicas de la Universidad Santo Tomás, Floridablanca
}

\author{
Cross-infection by composite resin tubes used in dental clinics in Universidad San- \\ to Tomás, Floridablanca
}

\author{
David Felipe Niño Duarte', Melissa Tatiana Niño Ortiz' , Paula Andrea Ortega Torres' , Jairo Andrés Peña Hernández' , Jairo \\ Fernando Picón Peña²
}

\begin{abstract}
Para citar este artículo: Niño DF, Niño MT, Ortega PA, Peña-Hernández JA, Picón-Peña JF. Contaminación cruzada por el uso de tubos de resinas
\end{abstract} compartidos en las clínicas odontológicas de la Universidad Santo Tomás, Floridablanca. Ustasalud 2016;15:12-19.

Licencia Creative Commons

\section{(c) (1) $(9 \Theta$} lo tanto, los lectores pueden acceder libremente a los artículos en su formato .pdf, igualmente podrán descargarlos y difundirlos; sin embargo no podrán modificarlos o alterarlos, adicionalmente se debe reconocer la autoría de las personas que figuran en las publicaciones, pero estas no podrán comercializadas.

\begin{abstract}
1 Facultad de Odontología, Universidad Santo Tomás, Bucaramanga.

${ }^{2}$ Grupo de Investigación Sistema Estomatognático y Morfofisiología - SEMF, Tecnología de Laboratorio Dental, Universidad Santo Tomás, Bucaramanga
\end{abstract}

Autor de correspondencia: Jairo Fernando Picón Peña

Correo electrónico:

jairof.picon@ustabuca.edu.co

\section{RESUMEN}

Objetivo: determinar la contaminación cruzada producida por el uso de tubos de resina condensable compartidos entre pacientes por los estudiantes en las Clínicas Odontológicas de la Universidad Santo Tomás Campus Floridablanca.

Materiales y métodos: estudio observacional In vitro, en el que se usaron 39 tubos de resina $3 \mathrm{M}$ del sector posterior de referencia (P60), con disponibilidad de los colores B2, A2 y A3 utilizados entre pacientes en las Clínicas Odontológicas de la Universidad Santo Tomás Campus Floridablanca, de séptimo a décimo semestre durante el segundo período del año 2014. Estos tubos están distribuidos: 13 por cada piso y se tomaron muestras de la resina que contenían para luego ser analizados mediante pruebas de laboratorio con el fin de establecer la presencia de microorganismos, además de identificar los microorganismos encontrados causantes de la contaminación.

Resultados: el 50\% de las muestras resultaron contaminadas con 15 microorganismos principales y tres secundarios.

Conclusión: el porcentaje de muestras contaminadas es significativo, lo que indica que existe contaminación al momento de manipular las resinas.

Palabras clave: resinas compuestas, microorganismos, pautas de la práctica en odontología.

\section{ABSTRACT}

Objective: to determine the cross-infection caused by resin tubes shared among patients on Dental Clinics at Universidad Santo Tomás, Floridablanca.

Materials and methods: In vitro observational study. 39 composite resin tubes for posterior teeth (P60) and manufactured by $3 \mathrm{M}$ were analyzed, the colors available were $\mathrm{B} 2, \mathrm{~A} 2$ and $\mathrm{A} 3$, used in patients at the Dental Clinics of Universidad Santo Tomás, manipulated by students from seventh to tenth semester during the second period of 2014. The resin tubes are distributed, 13 for each floor of the clinics and the samples were taken directly from the composite resin already in use, later the samples were analyzed by laboratory tests in order to establish the presence of microorganisms. Also, the microorganisms that could cause cross-infection were identified and finally a management scheme for these composite was proposed with the purpose to avoid contamination.

Results: $50 \%$ of the samples were contaminated with 15 main microorganisms and three secondary ones.

Conclusion: the percentage of contaminated samples of composite resins is significant, indicating that there is cross infection when handling the composite resins.

Keywords: composites, microorganisms, practice Patterns, Dentists. 


\section{INTRODUCCIÓN}

En el área de la salud en general, es importante seguir los protocolos y normas que se establecen basados en evidencia científica y de acuerdo con ciertas leyes (por ejemplo, la Ley 35 de 1989 o la Ley 9 de 1979), con el fin de ejecutar eficientemente un procedimiento con mayor éxito y sobre todo sin causar daño o riesgo para el paciente ${ }^{1}$.

La Asociación Dental Americana (ADA) ha sugerido, a través de los años, mejorar procedimientos para reducir la infección, debido a que en el área de la odontología es muy común la tendencia a presentarse infecciones por microorganismos presentes en la saliva que pueden causar enfermedades, tales como: tuberculosis, neumonía, herpes, hepatitis B y C, entre otras; esta vigilancia se hace a través de procedimientos eficaces, controlados y teniendo precauciones universales que pueden prevenir la infección cruzada. No se observa instrucciones específicas para la manipulación de resinas compuestas, ni procedimientos estándares para la desinfección del material sin causar ninguna alteración en su estructura ${ }^{2-6}$.

En las Clínicas Odontológicas de la Universidad Santo Tomás, los estudiantes durante los procedimientos operatorios comparten los tubos de resina, debido a que no se cuenta con la cantidad necesaria, ni la gama de colores completa para su uso individual. Por lo tanto, los estudiantes toman lo indicado para el procedimiento, utilizando espátulas para resina que hacen contacto con la boca del paciente y luego en los tubos de resina, que posteriormente se prestan a otros estudiantes de la misma clínica que hacen el mismo procedimiento. Además, podría existir un almacenamiento inadecuado de estas, causado por la falta de recipientes limpios y un posible desconocimiento del personal auxiliar sobre el mantenimiento óptimo de los materiales odontológicos. Esta situación hace posible la contaminación en esos tubos de resina que podría contribuir al fracaso de las restauraciones realizadas, generando caries secundaria y propagación de microorganismos ${ }^{2,5-8}$.

Una vez que la resina está contaminada, los microorganismos permanecen activos, incluso después de fotocurar el material; por lo tanto, la infección se convierte en un riesgo inminente para el paciente. Por otra parte, estos procedimientos odontológicos cuentan con normas de bioseguridad, almacenamiento y la desinfección adecuada, pero infortunadamente no se cumple por parte del estudiante ni del personal auxiliar, lo que hace que pueda existir una cadena potencial de infección cruzada de un paciente a otro, al igual que infección de un tubo de resina a otro. Además, no existe un protocolo específico para el manejo de los tubos resinas, el cual sería necesario para informar a los estudiantes sobre cómo evitar este tipo de contaminación y cómo realizar su correcta manipulación ${ }^{2-4,9}$.

El propósito de esta investigación es identificar si existe o no contaminación cruzada al compartir los tubos de resina entre pacientes en las Clínicas Odontológicas de la Universidad Santo Tomás, con el propósito de mejorar la calidad en la atención del servicio de la Institución y reducir los posibles fracasos de las restauraciones y la propagación de enfermedades infecciosas, además de velar por la salud del paciente.

\section{MATERIALES Y MÉTODOS}

Estudio observacional descriptivo in vitro, en el que se usaron 39 tubos de resina de la casa comercial $3 \mathrm{M}$ del sector posterior de referencia (P60), con disponibilidad de los colores B2, A2 y A3 utilizados entre pacientes en las Clínicas Odontológicas de la Universidad Santo Tomás Campus Floridablanca, de séptimo a décimo semestre durante el segundo período del 2014. Se distribuyeron 13 unidades en cada piso de las Clínicas. Este tamaño de muestra fue calculado por medio del programa Epi Info Versión 3.2.2 de 2004, subprograma Statcalc, en un $95 \%$ de confianza y $5 \%$ de error tipo 1, usando como referencia la presencia de contaminación con una prevalencia de un $51 \%$ de las resinas evaluadas ${ }^{3}$.

El tipo de muestreo fue no probabilístico por conveniencia; solo se usaron tubos de resinas condensables que se estuvieron utilizando de manera activa en las clínicas odontológicas por los estudiantes de pregrado, no se usaron tubos nuevos, ni tubos de resina que fuesen de propiedad de los estudiantes, ni tubos de resina fluida.

Para tomar las muestras se solicitó, previa autorización de la Dirección de Clínicas, a las auxiliares la entrega de los tubos de resinas utilizados en el transcurso 
de esa clínica. Antes de realizar la toma de la muestra se marcaron los tubos de recepción de muestras con la siguiente información: color de resina, piso de las clínicas, fecha de toma, referencia del tubo, muestra pre y pos. En el momento en el que el estudiante solicitó la resina, se realizó la toma de muestra pre, cortando dos porciones de $2 \mathrm{~mm}$ aproximadamente de resina de cada tubo (el primero fue desechado), en cada uno de los tres pisos de las Clínicas Odontológicas de la Universidad Santo Tomás Campus Floridablanca, para este proceso se utilizó una espátula de resina estéril para retirar el material restaurador del tubo y depositarlo en $3 \mathrm{ml}$ de caldo nutritivo (Tripticasa Soya), durante este proceso fue necesario flamear la boca del tubo para evitar la contaminación (Figura 1).
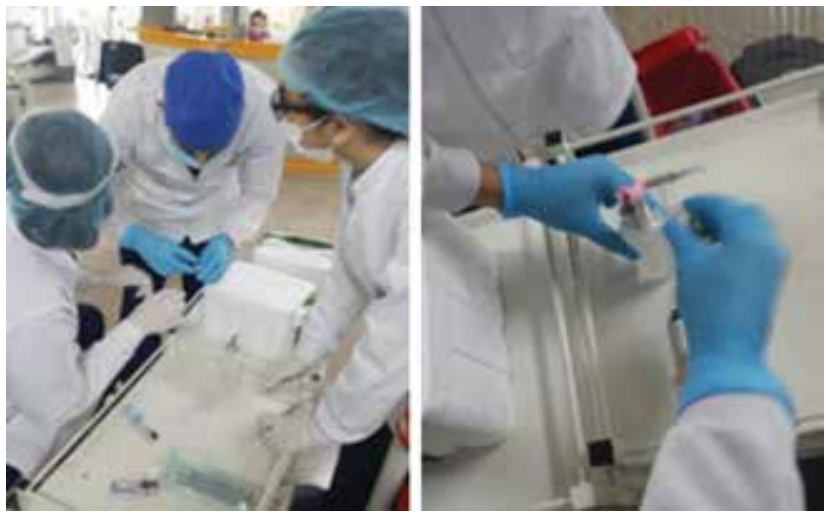

Figura 1. Toma de muestra de tubos de resina en las Clínicas de la Universidad Santo Tomás Campus Floridablanca, segundo piso.

Posteriormente se entregaron los tubos de resina a los estudiantes para que fueran usados durante la actividad asistencial, en permanente observación por parte de los investigadores, y luego de ser usados, los estudiantes regresaron el tubo de resina para proceder a tomar la muestra pos de igual forma que el pre.

Cada tubo de ensayo tapa rosca con la muestra de resina se tapó y se selló con cinta de enmascarar para evitar el derrame del caldo y del material, se ubicó en porta tubos y se almacenó en un termo limpio y desinfectado para trasladar las muestras al Laboratorio de Microbiología de la Universidad Industrial de Santander. Las muestras se llevaron al laboratorio en el menor tiempo posible. Las condiciones de toma y traslado fueron controladas, siguiendo las normas de bioseguridad, para evitar la posible contaminación con otros microorganismos o la destrucción de los mismos a partir del traslado ${ }^{4,10}$ (Figura 2).
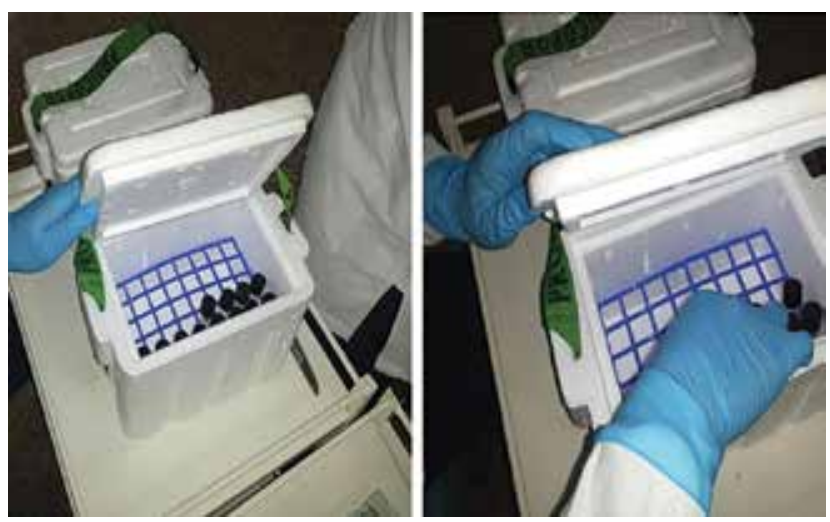

Figura 2. Almacenamiento de los tubos con la muestra para el envío al laboratorio.

Los tubos con las muestras se llevaron a la estufa incubadora a una temperatura de $37^{\circ} \mathrm{C}$ durante 24 a 48 horas, se dejó un tubo en cada muestreo sin inocular como control negativo. Se determinó la contaminación microbiana a través de la presencia de turbidez, un sedimento blanco o película superficial. Las muestras que cumplieron con estas características se repicaron en medios de cultivo selectivos para lograr el aislamiento de los microorganismos objeto de estudio 5 .

Luego de obtener el cultivo puro, en agar sangre para aislamiento de Staphylococcus sp y Streptococcus sp, en agar sabouraud para aislamiento de Candida albicans y agar MacConkey para aislar Escherichia coli se realizó una coloración de Gram para identificar la morfología y tinción de las bacterias, cocos y bacilos Gram (+) o Gram (-) y una coloración de azul de lactofenol para observar las levaduras características.

Posteriormente, se realizaron las pruebas confirmatorias como la detección de catalasa que permite diferenciar el género Stahylococcus sp y Escherichia coli (catalasa positivo) de los géneros Streptococcus $s p$ y Enterococcus $s p$ (catalasa negativos). La fermentación de glucosa permite diferenciar el género Staphylococcus sp del género Micrococcus sp, que es también catalasa positivo, pero no fermenta en glucosa en anaerobiosis ${ }^{4}$. 
La prueba de la coagulasa se utilizó para la identificación de Staphylococcus aureus, se basa en la capacidad de estas cepas para producir esta enzima extracelular que coagula el plasma, permite diferenciar al Staphylococcus aureus (coagulasa positivo) de todas las demás especies de Staphylococcus (coagulasa negativos). Para la Cándida albicans se utilizaron las pruebas de azul de lactofenol y se realizó la prueba de tubo germinal para establecer la especie albicans ${ }^{4}$.

La identificación de Streptococcus pyogenes se logró por la beta hemólisis que produce en el medio de cultivo de agar sangre así como la morfología de las colonias las cuales son usualmente secas y pequeñas con relación a la zona de hemólisis; adicionalmente, se emplearon antisueros anti- Strep A para la clasificación. Debido a que los resultados, con respecto a los anteriores microorganismos, dieron negativos, se usó el sistema BBL Crystal para la identificación de anaerobios (ANR). Este es un método de identificación en miniatura que utiliza substratos convencionales, fluorogénicos y cromogénicos modificados, los cuales se han diseñado para la identificación de bacterias anaerobias aisladas frecuentemente de muestras clínicas ${ }^{11}$.

\section{RESULTADOS}

El color de resina para el sector posterior más usado es el B2, el cual equivale al 61,5\%, seguido del A2 con un $35,9 \%$ y el A3 con 2,6\% (Tabla 1).
Tabla 1. Color de resina para el sector posterior presentes en los pisos de las Clínicas Odontológicas de la Universidad Santo Tomás

\begin{tabular}{ccc}
\hline Color & Frecuencia & Porcentaje (\%) \\
\hline A2 & 28 & 35,9 \\
B2 & 48 & 61,5 \\
A3 & 2 & 2,6 \\
Total & 78 & 100 \\
\hline
\end{tabular}

Un total de 78 muestras fueron obtenidas, 26 en cada uno de los tres pisos de la clínica. Con respecto al aislamiento microbiológico, el resultado negativo fue el más predominante con $50 \%$. En todos los pisos de las clínicas el microorganismo aislado con mayor frecuencia fue el Staphylococcus epidermidis (33\%). Bacillus subtilis, Staphylococcus saprophyticus, Corynebacterium spp y Staphylococcus warneri se encontraron unicamente en el segundo piso. El único aislamiento de Staphylococcus capitis se realizó en el cuarto piso. El cuarto piso también presentó el mayor número de muestras negativas $(n=15)$ y en el tercer piso fue en el que se observó mayor número de muestras con el Staphylococcus epidermidis $(\mathrm{n}=11)$ (Tabla 2).

El 33,3\% de los aislamientos correspondió a cada uno de los tres microorganismos secundarios presentes en las muestras tomadas de las Clínicas Odontológicas de la Universidad Santo Tomás (Tabla 3).

Tabla 2. Tabla resumen de la frecuencia de aislamiento de microorganismos por piso en las Clínicas Odontológicas de la Universidad Santo Tomás

\begin{tabular}{lcccc}
\hline Microorganismo aislado & $\begin{array}{c}\text { Frecuencia } \\
\mathbf{n}(\mathbf{\%})\end{array}$ & $\begin{array}{c}\text { Piso 2 } \\
\mathbf{n}(\mathbf{\%})\end{array}$ & $\begin{array}{c}\text { Piso 3 } \\
\mathbf{n}(\%)\end{array}$ & $\begin{array}{c}\text { Piso 4 } \\
\mathbf{n}(\mathbf{\%})\end{array}$ \\
\hline Ninguno & $39(50,0)$ & $12(46,2)$ & $12(46,2)$ & $\mathbf{1 5}(\mathbf{5 7}, 7)$ \\
Staphylococcus epidermidis & $26(33,3)$ & $6(23,1)$ & $11(42,3)$ & $9(34,6)$ \\
\hline Bacillus subtilis & $1(1,3)$ & $1(3,8)$ & $0(0,0)$ & $0(0,0)$ \\
Bacillus circulans & $4(5,1)$ & $3(11,5)$ & $1(3,8)$ & $0(0,0)$ \\
\hline Staphylococcus saprophyticus & $1(1,3)$ & $1(3,8)$ & $0(0,0)$ & $0(0,0)$ \\
\hline Streptococcus mitis & $2(2,5)$ & $0(0,0)$ & $1(3,8)$ & $1(3,8)$ \\
Staphylococcus hominis & $2(2,5)$ & $1(3,8)$ & $1(3,8)$ & $0(0,0)$ \\
Corynebacterium spp & $1(1,3)$ & $1(3,8)$ & $0(0,0)$ & $0(0,0)$ \\
\hline Staphylococcus warneri & $1(1,3)$ & $1(3,8)$ & $0(0,0)$ & $0(0,0)$ \\
\hline Staphylococcus capitis & $1(1,3)$ & $0(0,0)$ & $0(0,0)$ & $1(3,8)$ \\
Total & $\mathbf{7 8}(\mathbf{1 0 0})$ & $\mathbf{2 6}(\mathbf{1 0 0})$ & $\mathbf{2 6}(\mathbf{1 0 0})$ & $\mathbf{2 6}(\mathbf{1 0 0})$ \\
\hline
\end{tabular}


Tabla 3. Microorganismos secundarios presentes en la muestra

\begin{tabular}{lc}
\hline Microorganismo & Frecuencia n(\%) \\
\hline Micrococcus luteus & $1(33,3)$ \\
Staphylococcus intermedius & $1(33,3)$ \\
Staphylococcus salivarius & $1(33,3)$ \\
Total & $3(100)$ \\
\hline
\end{tabular}

Respecto al momento de la toma de la muestra, un $64,1 \%$ de las pruebas en la toma pre fueron negativas para un total de 25 muestras, mientras que en la muestra pos el Staphylococcus epidermidis representó el $38,5 \%$, seguido de la muestra pos con resultado negativo, la cual correspondió al 35,9\% del total de las muestras., El valor de $\mathrm{P}$ muestra que existe diferencia significativa entre la cantidad de muestras negativas y el momento de la toma (Tabla 4). En cuanto a los microorganismos presentes en cada uno de los pisos antes y después de los procedimientos con pacientes, no hubo diferencias estadísticamente significativas (piso $2 \mathrm{p}=0,395$; piso $3 \mathrm{p}=0,158$ y piso $4 \mathrm{p}=0,438$ ).

Tabla 4. Microorganismos presentes en el tubo de resina de las Clínicas Odontológicas de la Universidad Santo

Tomás respecto al momento de la toma

\begin{tabular}{|c|c|c|c|}
\hline \multirow{2}{*}{ Microorganismo } & \multicolumn{2}{|c|}{ Momento de la toma } & \multirow{2}{*}{ Valor de $\mathrm{P}$} \\
\hline & Pre $n(\%)$ & Pos n(\%) & \\
\hline Negativo & $25(64,1)$ & $14(35,9)$ & \multirow{11}{*}{0,013} \\
\hline Staphylococcus epidermidis & $11(28,2)$ & $15(38,5)$ & \\
\hline Bacillus subtilis & $0(0,0)$ & $1(2,6)$ & \\
\hline Bacillus circulans & $2(5,1)$ & $2(5,1)$ & \\
\hline Staphylococcus saprophyticus & $0(0,0)$ & $1(2,6)$ & \\
\hline Streptococcus mitis & $1(2,6)$ & $1(2,6)$ & \\
\hline Staphylococcus hominis & $0(0,0)$ & $2(5,1)$ & \\
\hline Corynebacterium spp & $0(0,0)$ & $1(2,6)$ & \\
\hline Staphylococcus warneri & $0(0,0)$ & $1(2,6)$ & \\
\hline Staphylococcus capitis & $0(0,0)$ & $1(2,6)$ & \\
\hline Total & $39(100)$ & $39(100)$ & \\
\hline
\end{tabular}

De los microorganismos secundarios, se observó que durante la muestra pre, el Micrococcus luteus fue el único microorganismo presente, mientras que en la muestra pos el Staphylococcus intermedius y Streptococcus salivarius representaron cada uno el 50\% de las muestras durante esta toma (Tabla 5). En cuanto el análisis pre y pos de los microorganismos secundarios identificados en cada uno de los pisos, no hubo diferencias estadísticamente significativas $(\mathrm{p}>0,05)$.
Tabla 5. Microorganismos secundarios presentes en las muestras con respecto al momento de la toma

\begin{tabular}{lcc}
\hline Microorganismo & \multicolumn{2}{c}{ Momento de la toma } \\
\cline { 2 - 3 } & Pre $\mathrm{n}(\%)$ & $\operatorname{Pos} \mathrm{n}(\%)$ \\
\hline Micrococcus luteus & $1(100)$ & $0(0,0)$ \\
Staphylococcus intermedius & $0(0,0)$ & $1(50)$ \\
Staphylococcus salivarius & $0(0,0)$ & $1(50)$ \\
Total & $\mathbf{1 ( 1 0 0 )}$ & $\mathbf{2 ( 1 0 0 )}$ \\
\hline
\end{tabular}

\section{DISCUSIÓN}

En el presente estudio se evidenció la presencia de microorganismos en las muestras de resina tomadas de los tubos que comparten los estudiantes en las Clínicas de la Universidad Santo Tomás, muestras que se obtuvieron antes y después de ser manipuladas.

Los resultados mostraron que solo el $50 \%$ del total de las muestras se clasificó como negativo, es decir, que no presenta contaminación de ningún microorganismo en las tomas pre y pos de las resinas.

El principal microorganismo que generó contaminación fue el Staphylococcus epidermidis, con una frecuencia de $33,3 \%$ en el total de las muestras, cuestión preocupante, ya que este microorganismo es generador de infecciones de varios tipos y presenta las características para poder multiplicarse fácilmente, formar biofilm y además alcanzar el torrente circulatorio $^{12}$.

El segundo microorganismo presente en las muestras fue el Bacillus circulans con un 5,1\% en las muestras de resina. Este microorganismo está presente en el medio ambiente, lo que demuestra que la contaminación que se produce puede ser causada también por el medio en el que se encuentran circulando las resinas ${ }^{13-15}$. Por otra parte, el Staphylococcus hominis tuvo una frecuencia del $5,1 \%$ en las muestras, este es un microorganismo que tiende a adquirirse de forma nosocomial, lo que dice que es una cuestión relacionada más al medio ambiente de las clínicas que a la manipulación de los tubos entre pacientes, pero cabe aclarar que es algo que requiere atención al tratarse de un microorganismo que puede llegar a ser perjucidial en personas comprometidas inmunológicamente por alguna enfermedad o por quimioterapia ${ }^{16,17}$. 
De igual forma, existió contaminación con el microorganismo Streptococcus mitis que estuvo presente en dos muestras $(2,6 \%)$. Este microorganismo es comensal de la cavidad oral y hace parte de la formación de la biopelícula dental que podría llevar a una infección odontogénica. Aquí es evidente que la contaminación es debida al contacto de la resina con la boca, como consecuencia de una inadecuada manipulación y desinfección, ya que el microorganismo resultó en el cultivo de una muestra pre y una pos ${ }^{18,19,20,21}$. Por otra parte, existieron tres muestras con segundo microorganismo, uno en la toma pre y dos en la toma pos, estos fueron el Micrococcus luteus, Staphylococcus intermedius y el Streptococcus salivarius, siendo este último junto al Streptococcus mitis, los dos únicos que se encuentran en la microbiota oral ${ }^{18-21}$.

En el estudio de Werle y colaboradores, (2012), los resultados demostraron contaminación de más del $50 \%$ en todas las muestras tomadas, y es un resultado preocupante, ya que se realizó en una institución en la cual se presentan todos los decretos y las normas de bioseguridad establecidas por la agencia de vigilancia sanitaria. En esta investigación se propone como solución la utilización de un método de dispensación por medio del uso de un vaso dapen, para así evitar la contaminación entre pacientes por el uso compartido de los tubos de resina por parte de los estudiantes, pero también denota que es un método difícil de implementar, debido a que es más complicado para el estudiante y para las personas que deban dispensar el material ${ }^{2}$. Adicionalmente, dice que la contaminación estuvo presente en 2 de los 5 grupos que evaluaron, representado un $46,9 \%$ del total de las muestras ${ }^{2}$.

En el estudio realizado por Ventura-Egusquiza (2006) se logró demostrar la contaminación que se encuentra en las clínicas odontológicas a las que asisten los estudiantes y concluye que la contaminación cruzada es un proceso muy factible y que se produce debido al medio, el divide en 5 los puntos más críticos a poder contraer y proveer contaminación cruzada, $y$ en primer lugar con un riesgo alto están las jeringas triples, lo que podría ser preocupante, ya que se deben usar constantemente en la atención odontológica y por medio de ellas es que se lava y se irrigan las cavidades por restaurar, pudiendo entonces demostrarse que la cavidad se contamina directamente no por la resina sino por el agua usada para su lavado ${ }^{4}$.
Pedrosa y colaboradores, (2010) determinaron el grado de contaminación en 55 tubos de resina compuesta de varios consultorios odontológicos en Brasil. Revelaron que el $80 \%$ se encontraban contaminadas con Staphylococcus coagulasa - negativa, Bacillus $s p$ y Aspergillus sp y demostraron una inadecuada manipulación de los tubos de resina compuesta por parte de los odontólogos y auxiliares ${ }^{22}$.

Cardoso y colaboradores, (2010) en la investigación "Contaminação de tubos de resina composta manipulados sem barreira de proteção" evaluaron la contaminación de la parte externa de los tubos de resina, realizaron un recuento total de microrganismos viables y se reportó una cantidad elevada del género Staphylococcus ${ }^{23}$.

Oliveira y colaboradores, (2010) analizaron la contaminación bacteriana en 50 tubos de resina compuesta utilizada en la clínica de la Facultad de Odontología de la Universidad Federal de Juiz de Fora. En sus resultados observaron que solo 2 de las resinas compuestas usadas por los estudiantes de la clínica presentaron contaminantes biológicos, esto representa solo el $4 \%$ del total de las muestras evaluadas, concluyendo que las técnicas de mantenimiento de la cadena aséptica y las normas de bioseguridad empleadas en las clínicas de la Facultad son eficaces ${ }^{24}$.

A nivel nacional, en el 2016, un estudio realizado en la Facultad de Odontología de la Universidad Cooperativa de Colombia, sede Envigado, manifestó que el $34,8 \%$ de 46 muestras de resina, estaban contaminadas por bacterias del género Staphylococcus y bacilos Gram positivos. Sin evaluar la presencia del microorganismo antes y después de su uso, comprobaron que "con sólo un uso de la resina después del procedimiento clínico en un paciente es suficiente para promover su contaminación, por lo tanto el remanente de la resina puede ser considerado como uno de los vehículos responsables de contaminación cruzada”25.

Este estudio se enfocó en la manipulación de los tubos de resina por parte de los estudiantes, pero observando los resultados es claro que hubiese sido necesario evaluar los procesos de almacenamiento y manejo por parte de las auxiliares de las clínicas, situación en la que también puede existir contaminación, lo que explicaría de mejor forma los microorganismos 
encontrados en las pruebas pre. De esta manera, las inquietudes que derivan de este proyecto consisten en la explicación de la contaminación encontrada en las muestras antes de ser usadas por el estudiante y la evaluación de medidas que disminuyan este tipo de contaminación cruzada.

\section{CONCLUSIÓN}

El 50\% de las muestras presentaron contaminación microbiológica. Analizando el momento de la toma, todos los microorganismos aumentaron su presencia en las muestras analizadas después de ser manipuladas por el estudiante, lo que indica que existen fallos en la manipulación que se le da a los tubos de resina y que el hecho de compartirlos puede generar contaminación cruzada. De igual manera preocupa la contaminación presente en las muestras pre, que da a entender problemas en el almacenamiento o en la desinfección de los tubos. Lo anterior además es indicador de uso inadecuado de las barreras de bioseguridad y contaminación presente en el medio ambiente de las clínicas ya que los microorganismos que se aislaron en las muestras, se encuentran en el ambiente y en la piel.

\section{BIBLIOGRAFÍA}

1. Barco Virgilio, Díaz Uribe Eduardo. Código de Ética del Odontólogo Colombiano Ley 35 de 1989 Tribunal de Ética Odontológica Seccional Valle del Cauca Cali [Internet] Recuperado a partir de: http://medicinaprepagada.coomeva.com.co/archivos/odon/codigo_etica.pdf

2. Werle S, Vianna R, Santos, Dotto P. Contamination of composite resins in dental clinics of a teaching institution. Pesq Bras Odontoped ClinIntegr. 2012;12(4):473-476.

3. Ferraz C, Rocha C, Rocha M, Martins M, Jacques P. Contamination of resin composites in dental practice. Pesq Bras Odontoped Clin Integr. 2010;10(1):73-78.

4. Ventura Egusquiza CD. Grado de contaminación cruzada en la atención de la clínica $\mathrm{N}^{\circ} 1$ de la Facultad de Odontología de la Universidad Nacional Mayor de San Marcos mediante un indicador biológico [tesis], [Lima - Perú], [Universidad Nacional Mayor de San Marcos] 2006.

5. Alfonso Mariana PB. Riesgo de contaminación cruzada para el paciente que asiste a las clínicas de la Facultad de Odontología de la Universidad Francisco Marroquín, 2000, [tesis], [Guatemala], [Universidad Francisco Marroquín], 2001.
6. $3 \mathrm{~m}^{\mathrm{Tm}}$ Filtek ${ }^{\mathrm{Tm}}$ P60 Restaurador Posterior. Perfil técnico del producto. Págs. 34-37. 3M.

7. Moncada G, Fernández E, Martin J, Caro MJ, Caamaño C, Mjor I, Gordan V. Longevidad y causas de fracaso de restauraciones de amalgama y resina compuesta. Revista Dental de Chile. 2007;99(3);8-16.

8. Fakhri M, Seraj B, Shahrabi M, Motahhary P, Hooshmand T. Efecto de la contaminación con la saliva en la microfiltración de las resinas compuestas colocado con adhesivo de autograbado en la dentición temporal: un estudio in vitro. Odontología Pediátrica. 2009;31(4):334-339.

9. Comité de Infecciones y Vigilancia Epidemiológica y Direcciones Clínicas Odontológicas. Manual básico de procedimientos de bioseguridad para clínicas odontológicas de la IPS Universidad Santo Tomás [Biblioteca USTA] (Versión 3 enero de 2013).

10. Organización Mundial de la Salud. Manual de bioseguridad en el laboratorio. 3era edición. Ginebra. OMS, 2005.

11. Sistemas de Identificación BBL Crystal. Equipo para la identificación de anaerobios. Manual de uso sistema BBL crystal.

12. Koneman E, Allen S. Diagnóstico microbiológico. 3era. edición. Editorial Médica Panamericana. Buenos Aires, Argentina; 2008.

13. Abis Enciclopedia. Bacillus circulans. [Internet] Recuperada a partir de: http://www.tgw1916.net/Bacillus/circulans.html.

14. Rodríguez G. Géneros Streptococcus y Enterococcus. 2da. edición. Uruguay Editorial Médica. 2006.

15. Diccionario de enfermedades. Bacillus circulans. Recuperada a partir de: http://www.ayudin.com.ar/diccionario_ resultados.php? $\mathrm{id}=74 \&$ tipo=germenes\&letra $=\mathrm{B} \& p g=1$ (19 noviembre 2014).

16. Bacteriemia nosocomial por Staphylococcus hominis. Coria JJ, Mora R, Pérez VM, De la Cruz R, Vázquez A. Revista de Enfermedades Infecciosas en Pediatría. Vol. XXIII Núm. 91. Enero - marzo 2010. Recuperada a partir de: http://www.medigraphic.com/pdfs/revenfinfped/eip2010/eip101e.pdf

17. Coria JJ, Mora R, Pérez VM, De la Cruz R, Vázquez A, Bacteriemia nosocomial por Staphylococcus hominis, brote en la unidad de cuidados intensivos neonatales de un hospital de alta especialidad. Revista Enfermedades Infecciosas en Pediatría [Internet]. Enero. 2010;22/12/14:8792. Recuperada a partir de: http://www.medigraphic. com/pdfs/revenfinfped/eip-2010/eip101e.pdf. 
18. Negroni M. Microbiología estomatológica. Buenos Aires [etc.]: Médica Panamericana; 2009. 2ºdición, [pag. 400].

19. Public Health Agency of Canadá, Streptococcus salivarius - Ficha de seguridad - sustancias infecciosas, Public Health Agency of Canadá, [Internet] 2011, 19 noviembre 2014, Recuperada a partir de: http://www.phac-aspc.gc.ca/lab$\mathrm{bio} / \mathrm{res} / \mathrm{psds}$-ftss/streptococcus-salivarius-eng.php

20. Montiel AF, Flora bacteriana habitual, Boletín de la Escuela de Medicina Chile [Internet]. Marzo de 1997;22/12/14:15. Recuperada a partir de: http://escuela.med.puc.cl/publ/ boletin/laboratorio/florabacteriana.html

21. Mantzourani M, Gilbert SC, Fenlon M, and Beighton D. Non-oral bifidobacteria and the aciduric microbiota of the denture plaque biofilm. Molecular Oral Microbiology. 2010;25:190-199.
22. Pedrosa S, Moura MA, Chaves RM et al. Contaminação de resinas compostas em consultórios odontológicos. Rev Odontol Bras Central. 2010;19(50):211-15.

23. Cardoso CT, Pinto JR, Pereira EA, Barros LM, Freitas AB. Contaminação de tubos de resina composta manipulados sem barreira de proteção. Rev Odontol Bras Central. 2010;18(48):71-5.

24. Oliveira M, Barreto RM, Salgado IO, Chaves HD, Diniz CG. Avaliação da contaminação bacteriana em resinas compostas utilizadas nas clínicas de graduação da FO-UFJF. Odontol. Clín.-Cient. 2010;9(1):73-6.

25. Bedoya C, Sarrazola Á, Palacio S, Julio O, Osorio N, Garzón A. Evaluación de la contaminación microbiana en las resinas de fotocurado utilizada por estudiantes de odontología en sus prácticas clínicas. Estomatol. 2016;24(1):24-29. 